\title{
Digital Image Filtering in Transform Domain using MATLAB
}

\author{
R. Swaminathan \\ Asst. Professor, \\ Department of Computer Science \\ Urumu Dhanalakshmi College,Kattur, \\ Tiruchirappalli - 620019, \\ Tamil Nadu, \\ India.
}

\author{
T. Meyyappan, $\mathrm{PhD}$ \\ Professor,Department of Computer Science and \\ Engineering, Alagappa University, \\ Karaikudi - 630003 \\ Tamil Nadu, \\ India.
}

\begin{abstract}
Application of filter on digital images can be made in two ways, which include spatial domain and transform domain known as frequency domain. The spatial domain deals directly with manipulation of data, pixel, present in an image, whereas the transform domain deals with manipulation of image-data in frequency domain. The aim of this paper is to deal with manipulation of data present in an image in frequency domain and identification of performance of frequency domain low-pass filters in terms of removing noise present in the digital image and frequency domain high-pass filter in terms of highlighting the edge of the digital image. And this paper also deals with image-quality measuring tools such as MSE and PSNR for the purpose of identifying a frequency domain low-pass filter which is best at removing salt and pepper noise present in the digital image.
\end{abstract}

\section{Keywords}

Spatial domain, Frequency domain, Transform domain, Ideal low-pass filter, Ideal high-pass filter, Butterworth filter and Gaussian filter.

\section{INTRODUCTION}

Spatial domain and Transform domain are the methods in which filters can be applied on digital images. The purpose of filter is to enhance the details of an image by choosing or rejecting certain frequent components present in it. The spatial domain method operates directly on pixels, whereas the transform domain method operates on the Fourier transform of an image and then transforms it back to the spatial. [1] A symbolic representation for filtering in both the spatial and frequency domain is given below

$$
f(x, y) * h(x, y) \Leftrightarrow H(u, v) F(u, v)
$$

The expression indicates that convolution of two spatial functions can be obtained by computing the inverse Fourier transform of the product of the Fourier transform of the two functions. In the above symbolic representation, $H(u, v)$ is referred to as a filter transfer function and $F(u, v)$ is referred to as input image in Fourier transform [2]. The frequency domain filtering process can be thought of as a frequency domain mask, similar to spatial domain mask, and can be applied to Fourier transforms. And frequency domain filtering is attractive compared to spatial domain filtering because of fewer computations involved. This is because convolution in spatial domain is equivalent to multiplication in frequency domain. For smaller masks up to $9 \times$ 9, spatial domain is effective, but for larger masks, filtering in the frequency domain is preferred [1]. Therefore, this paper deals with digital image filtering in frequency domain. To convert an image from spatial domain to frequency domain, Fourier transform is being used. The 2D Fourier Transform is an important image processing tool to decompose a grayscale image into its sine and cosine components. The output of the transformation represents the image in the frequency domain [3].

In ref [4], the author has said that the high-pass filter preserves the edge details and the low-pass filter removes noise in an image by preserving details, and the Gaussian filter has minimum RMSE and maximum PSNR values. But, in this paper not only objective fidelity criteria but also subjective fidelity criteria have been used to identify the performance of filters. Its result has been shown in the section 4.1.1 and 4.2.

In ref [5], the author has used the same cut-off frequency for low-pass and high-pass filters and pointed out that higher order Butterworth low pass filter gives better smoothing result than lower order filter, Gaussian low pass filter's performance is better than lower order BLPF and the result of GHPF is similar to lower order Butterworth filter. But in this paper, two different cut-off frequencies have been used for the purpose of identifying the performance of low-pass and highpass filters.

In ref [6], the author has proposed a decision-based, detailpreserving restoration method and said that it is the ultimate filter for removing salt and pepper noise. But in this paper the removal of salt and pepper noise has been carried out using frequency domain filters.

In ref [7], the author has pointed out that High pass filtered images are very dark and as the cut-off frequency increases, the sharpness of the image also decreases. The outputs of the high-pass filters shown in this paper have the similar effect.

In ref [8], the author has carried out the removal of salt and pepper noise in digital images using spatial domain and said that the performance of median filter in removing salt and pepper noise in an image is better than wiener filter. But in this paper, the removal of salt and pepper in digital image has been carried out using transform domain.

\section{LOW-PASS FILTER}

A low-pass filter is a filter that allows low-frequency components and attenuates all other frequency components higher than the cut-off frequency. The actual amount of attenuation for each frequency varies depending on specific filter design. Smoothing is fundamentally a low-pass operation in the frequency domain [4]. There are various types of low-pass filter, which include Ideal low-pass filter, Butterworth low-pass filter and Gaussian low-pass filter. 


\subsection{Ideal low-pass filter}

An ideal low-pass filter allows all frequencies within the cutoff frequency $\mathrm{D}_{\mathrm{O}}$ and removes all other frequencies. Its transfer function is given below

$$
H(u, v)=\left\{\begin{array}{l}
1 \text { if } D(u, v) \leq D_{0} \\
0 \text { if } D(u, v)>D_{0}
\end{array}\right.
$$

Where $\mathrm{D}(\mathrm{u}, \mathrm{v})$ represents

$$
D(u, v)=\left[\left(u-\frac{m}{2}\right)^{2}+\left(v-\frac{N}{2}\right)^{2}\right]^{\frac{1}{2}}
$$

[1] In the above notation, the value of $\mathrm{H}(\mathrm{u}, \mathrm{v})$ becomes 1 if the value of $\mathrm{D}(\mathrm{u}, \mathrm{v})$ is lesser than $\mathrm{D}_{0}$. Otherwise the value of $\mathrm{H}(\mathrm{u}, \mathrm{v})$ becomes 0 .

\subsubsection{Implementation}

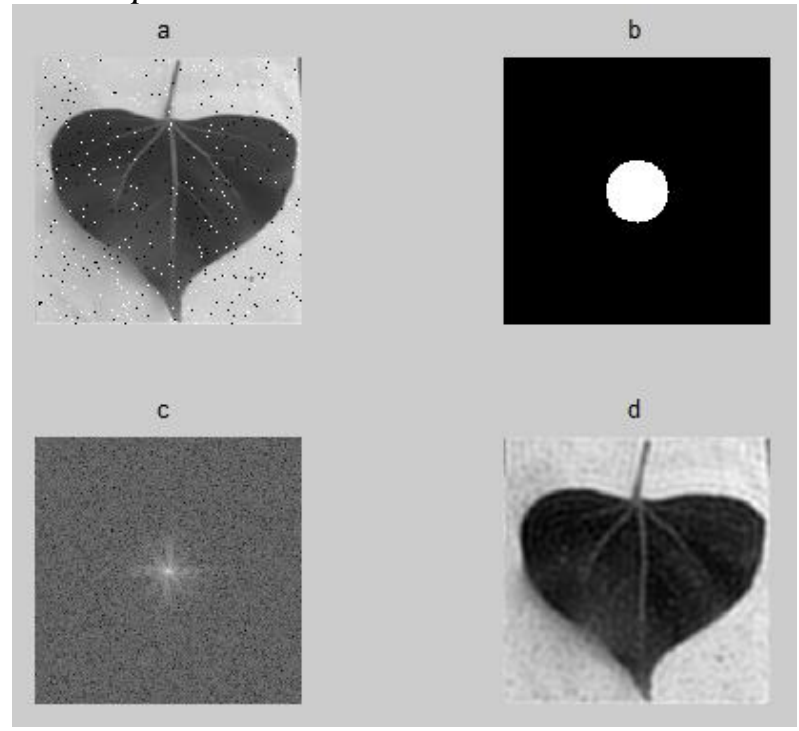

Fig -1: a) Image corrupted by salt and pepper noise withdensity 0.02 b) Ideal low-pass filter $\left[D_{0}=30\right]$ c) Spectrum of the original image (a). d) Filtered image

\subsection{Butterworth low-pass filter}

Butterworth low-pass filter is an effective filter in reducing or eliminating the ringing artifacts. Its transfer function is given below

$$
H(u, v)=\frac{1}{1+\left[\frac{\mathrm{D}(\mathrm{u}, \mathrm{v})}{\mathrm{D}_{0}}\right]^{2 \mathrm{n}}}
$$

In the above function, $\mathrm{n}$ is the order of the filter, $\mathrm{D}_{0}$ is the cutoff frequency and $\mathrm{H}$ is the magnitude of the filter mask and it has values range from 0 to 1 .

\subsubsection{Implementation}

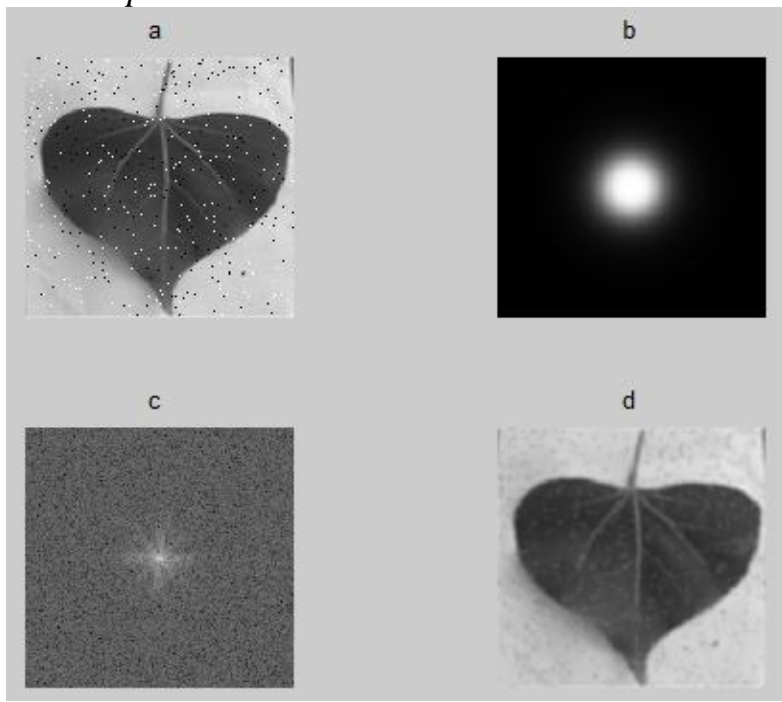

Fig -2 : a) Image corrupted by salt and pepper noise withdensity $0.02 \mathrm{~b}$ ) Butterworth low-pass filter $\left[\mathrm{D}_{0}=30\right] \mathrm{c}$ )

Spectrum of the original image (a). d) Filtered image

\subsection{Gaussian low-pass filter}

The Gaussian filter is useful for removing ringing and noise leakage artifacts. Its transfer function is given below

$$
H(u, v)=e^{\frac{-D^{2}(u, v)}{2 \sigma^{2}}}
$$

A transfer function is given below for cut-off frequency $\mathrm{D}_{0}$, which means $\sigma=\mathrm{D}_{0}$,

$$
H(u, v)=e^{\frac{-D^{2}(u, v)}{2 D_{0}{ }^{2}}}
$$

A change in the value of $\sigma$ will cause a similar effect in the cut-off frequency.

\subsubsection{Implementation}

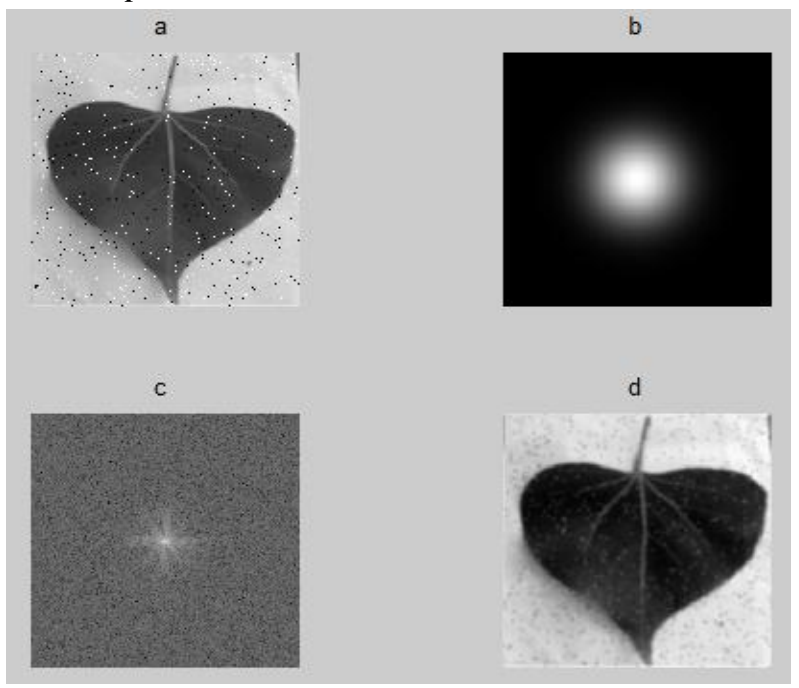

Fig -3: a) Image corrupted by salt $\&$ pepper noise withdensity 0.02 b) Gaussian low-pass filter $\left[D_{0}=30\right] c$ ) Spectrum of the original image (a). d) Filtered image

\section{HIGH-PASS FILTER}

A high-pass filter attenuates all low frequency components and allows all high frequency components such as edges, 
boundaries and other sudden changes of an image [1]. The transfer function of a high-pass filter can be designed as

$$
H_{h p}(\mathrm{u}, \mathrm{v})=1-\mathrm{H}_{\mathrm{lp}}(\mathrm{u}, \mathrm{v}) \quad[1][2]
$$

$\mathrm{H}_{\mathrm{hp}} \rightarrow$ Transfer function of high-pass filter

$\mathrm{H}_{\mathrm{Ip}} \rightarrow$ Transfer function of low-pass filter

\subsection{Ideal high-pass filter}

An ideal high-pass filter allows all frequencies components higher than the cut-off frequency $D_{O}$ and removes all other frequency components. Its transfer function is given below

$$
H(u, v)=\left\{\begin{array}{l}
0 \text { if } D(u, v) \leq D_{0} \\
1 \text { if } D(u, v)>D_{0}
\end{array}\right.
$$

The above transfer function states that it is opposite to the ideal low-pass filter.

\subsubsection{Implementation}

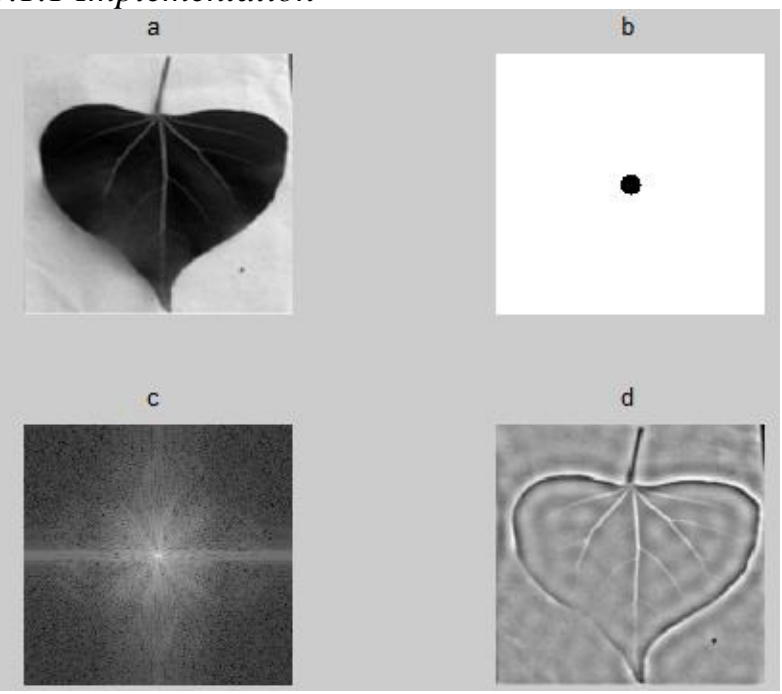

Fig -4: a) Original Image b) Ideal high-pass filter $\left.\left[D_{0}=10\right] c\right)$ Spectrum of the image(a), d)Filtered image

\subsection{Butterworth high-pass filter}

Butterworth high-pass filtering follows the process, which is opposite to the Butterworth low-pass filtering. The following is the transfer function of Butterworth high-pass filter.

$$
H(u, v)=\frac{1}{1+\left[\frac{\mathrm{D}_{0}}{\mathrm{D}(\mathrm{u}, \mathrm{v})}\right]^{2 \mathrm{n}}}
$$

In the above function, $\mathrm{n}$ plays an important role in determining the sharpness of cut-off frequency and ringing effect.

\subsubsection{Implementation}

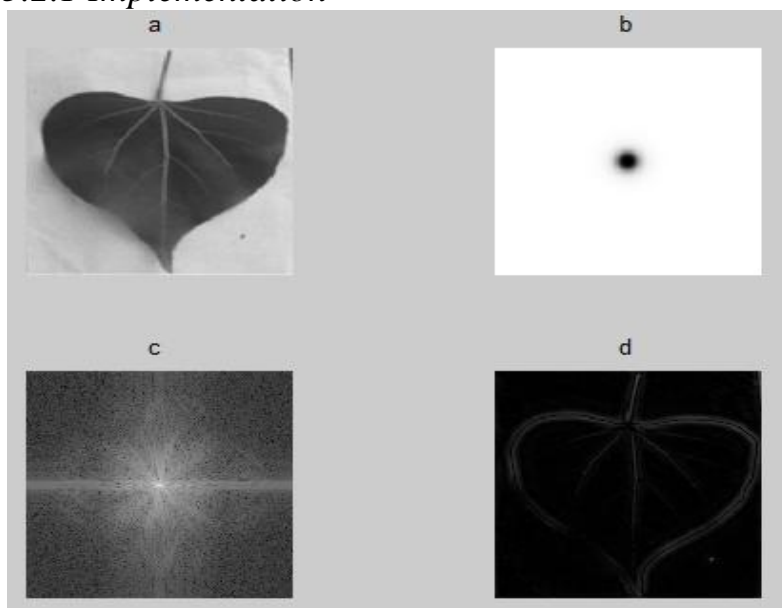

Fig -5: a) Original Image b)Butterworth high-pass filter $\left[D_{0}=10\right]$ c) Spectrum of the image $(a)$, d)Filtered image

\subsection{Gaussian high-pass filter}

The transfer function of Gaussian high-pass filter can be derived by subtracting the transfer function of Gaussian lowpass filter from 1 , which is stated below

$$
H(u, v)=1-e^{\frac{-D^{2}(u, v)}{2 D_{0}^{2}}}[1][2]
$$

\subsubsection{Implementation}

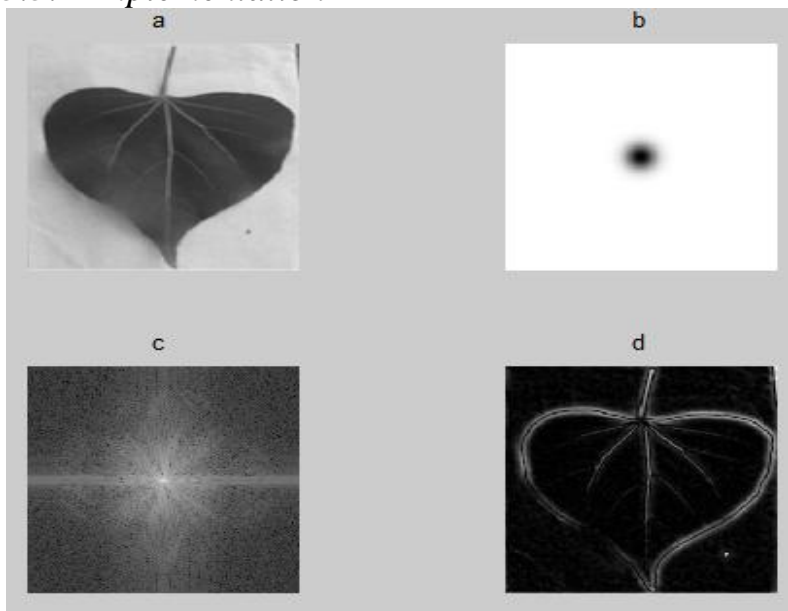

Fig -6: a) Original Image b) Gaussian high-pass filter $\left[D_{0}=10\right]$ c) Spectrum of the image(a),d)Filtered image

\section{IDENTIFICATION OF PERFORMANCE OF FILTERS}

This section comprises the outcome of low-pass and high-pass filters.

\section{1 Outcome of low-pass filters}

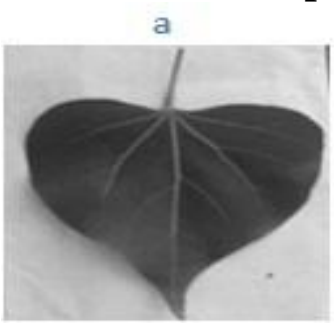

b

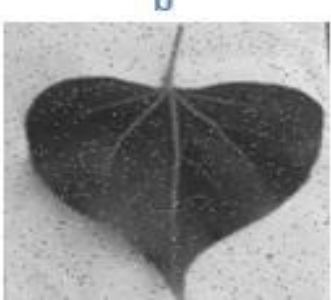



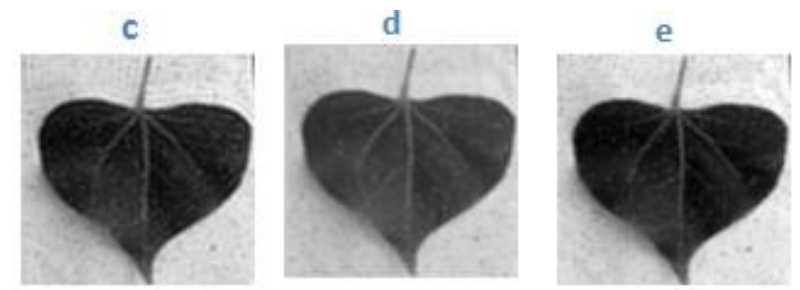

Fig -6: a) Original Image b) Image corrupted by salt $\&$ pepper noise $($ Density=0.02) c) Outcome of Ideal low-pass filter d) Outcome of Butterworth low-pass filter e) Outcome of Gaussian low-pass filter

\subsubsection{Image-Quality measuring tool}

This paper has used the following tools to calculate mean square error(MSE) and peak signal-to-noise ratio(PSNR) in order to characterize the quality of images produced by the filters, which include Ideal, Butterworth and Gaussian lowpass filters

$$
\begin{gathered}
M S E=\frac{1}{M N} \sum_{i=0}^{M-1} \sum_{j=0}^{N-1}[f(x, y)-\hat{f}(x, y)]^{2} \\
P S N R=20 \log _{10} \frac{255^{2} M N}{\sum_{i=0}^{M-1} \Sigma_{j=0}^{N-1}[f(x, y)-\hat{f}(x, y)]^{2}} d B \\
f(x, y) \text { - Original Image } \hat{f}(x, y)-\text { Filtered image }
\end{gathered}
$$

Table-1: MSE \& PSNR

\begin{tabular}{|l|c|c|c|}
\hline Image quality & \multicolumn{3}{|c|}{ FILTERS } \\
\cline { 2 - 4 } Measuring tool & ILPF & BWLPF & GLPF \\
\hline MSE & 115.17 & 21.12 & 119.41 \\
\hline PSNR & 55.10 & 69.83 & 54.78 \\
\hline
\end{tabular}

\subsection{Outcome of high-pass filters}
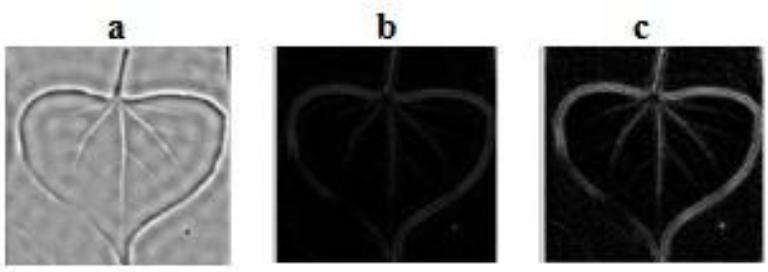

Fig -7 : a) Outcome of Ideal high-pass filter b) Outcome of Butterworth high-pass filter e) Outcome of Gaussian highpass filter

\section{CONCLUSION}

In this paper, objective and subjective fidelity criteria have been used for the purpose of measuring the quality of images produced by filters. Object fidelity criteria are based on metrics such as MSE, PSNR and so on. But, subjective fidelity criteria are based on human observer and not based on any metrics. And in this paper, object fidelity criteria have been used for measuring the quality of images produced by low-pass filters which have the same cut-off frequency $\left[D_{0}=30\right]$ whereas subject fidelity criteria have been used for measuring the quality of images produced by high-pass filters which have the same cut-off frequency $\left[\mathrm{D}_{0}=10\right]$. As per the information provided in the Table-1, the Butterworth low-pass filter is good in terms of removing salt \& pepper, because the image produced by the filter has low MSE(mean square error) and high PSNR (peak signal-to-noise-ratio). It is clear evident from the fig: 7 that the Ideal high-pass filter produces ringing effect, Butterworth high-pass has not highlighted the edges of the image clearly and Gaussian high-pass filter is best in terms of highlighting the edges of the image but darkens the image compared to Ideal and Butterworth high-pass filters.

\section{FUTURE SCOPE}

This paper deals only with grey scale images. In future, this research work can be extended by identification of performance of the filters mentioned in this paper by applying them on True-colour and Pseudo-colour images

\section{REFERENCES}

[1] Digital Image Processing, S. Sridhar, OXFORD University press.

[2] Digital Image Processing Using MATLAB, Second Edition, Rafael C. Gonzalez, Richard E. Woods, Steven L. Eddins, McGraw Hill Education(India) Private Limited.

[3] Frequency Domain Filtering of Colour Images using Quaternion Fourier Transforms,B.D.Venkatramana Reddy, Dr.T.Jayachandra Prasad, IJCST Vol. 1, Issue 2, December 2010, ISSN: 2229-4333(Print) ISSN : 09768491 (Online ).

[4] Image Enhancement Techniques using Highpass and Lowpass Filters, Aziz Makandar, Bhagirathi Halalli, International Journal of Computer Applications (0975 8887)Volume 109 - No. 14, January 2015.

[5] Analysis of Digital Image Filters in Frequency Domain,Md. Shahnawaz Shaikh,Ankita Choudhry,Rakhi Wadhwani,International Journal of Computer Applications (0975 - 8887)Volume 140 - No.6, April 2016

[6] Salt-and-Pepper Noise Removal by Median-Type Noise Detectors and Detail-Preserving Regularization, Raymond H. Chan, Chung-Wa Ho, and Mila Nikolova, IEEE TRANSACTIONS ON IMAGE PROCESSING, VOL. 14, NO. 10, OCTOBER 2005.

[7] Practical Digital Image Enhancements using Spatial and Frequency Domains Techniques, Babawuro Usman,Shehu Ayuba, International Research Journal of Computer Science (IRJCS) ISSN: 2393-9842, Issue 5, Volume 2 (May 2015).

[8] Performance Comparison of Median and Wiener Filter in Image De-noising,Suresh Kumar1, Papendra Kumar2, Manoj Gupta3, Ashok Kumar Nagawat4,International Journal of Computer Applications (0975 - 8887) Volume 12- No.4, November 2010. 